\title{
Second-Order Hierarchical Fast Terminal Sliding Model Control for a Class of Underactuated Systems Using Disturbance Observer
}

\author{
Wei Liu, Siyi Chen", Huixian Huang \\ School of Information Engineering, Xiangtan University, Xiangtan, China \\ Email address: \\ c.siyi@xtu.edu.cn (Siyi Chen) \\ ${ }^{*}$ Corresponding author
}

To cite this article:

Wei Liu, Siyi Chen, Huixian Huang. Second-Order Hierarchical Fast Terminal Sliding Model Control for a Class of Underactuated Systems Using Disturbance Observer. Automation, Control and Intelligent Systems. Vol. 7, No. 2, 2019, pp. 65-78. doi: 10.11648/j.acis.20190702.12

Received: April 15, 2019; Accepted: May 23, 2019; Published: June 15, 2019

\begin{abstract}
A second-order hierarchical fast terminal sliding mode control method based on disturbance observer (DOSHFTSM) is proposed for a class of fourth-order underactuated systems. In the first step, the fourth-order underactuated system is divided into two subsystems, and the integral sliding surface is designed for each subsystem. Then, the first-order fast terminal sliding surface is defined by using the integral sliding surface and its derivatives of each subsystem, and the switching control items of the system are designed according to the first-order fast terminal sliding surface of the subsystem. Secondly, the second-order sliding surface is designed by using the first-order fast terminal sliding surface of each subsystem. On the premise of ensuring the stability of Lyapunov, the switching control term is designed by using the variable coefficient double power reaching law to eliminate the system jitter. Finally, based on the principle of hyperbolic tangent nonlinear tracking differentiator, a hyperbolic tangent nonlinear disturbance observer (TANH-DOC) is designed to estimate the uncertainties and external disturbances of the system and compensate them to the sliding mode controller to improve the robustness of the system. The stability of the system is proved by using Lyapunov principle. The validity of this method is verified by numerical simulation and physical simulation of inverted pendulum system.
\end{abstract}

Keywords: Underactuated System, Disturbance Observer, Hierarchical Sliding Mode, Double Power Reaching Law, Stability Analysis

\section{Introduction}

In recent years, the public pay more and more attention to the improvement of underactuated systems. It usually appears in mechanical systems where the actuator is less than the control degree of freedom. It is widely applied in space robots, underwater robots, structural flexible robots, bridge cranes and other practical systems, and has published many papers on underactuated system control [1-12]. In a nutshell, controller design and stability analysis of nonlinear underactuated dynamic systems have always been an important research field.

Sliding mode control embraces premium robustness to system matching uncertainties and modeling errors. It is a kind of nonlinear control method with great significance that is widely used in various kinds of under actuation [13-17].
But, the traditional sliding mode control method makes the system state gradually converge to the equilibrium point on the sliding mode surface, which makes it difficult to achieve convergence in a finite time. So, terminal sliding model (TSM) greatly improves the convergence speed of the system near the equilibrium point by introducing terminal attractors [18-21]. However, in the traditional TSM, when the system state approaches the equilibrium state, the convergence speed of the nonlinear sliding mode is slower than that of the linear sliding mode. Therefore, a fast terminal sliding mode (FTSM) is proposed, which not only introduces the terminal attractor to make the system state converge in a limited time, but also retains the fast convergence of linear sliding mode when approaching the equilibrium state, thus realizing the fast and accurate convergence of the system state to the equilibrium state [22-27]. However, a large control signal 
will be generated probably in the steady state and there will be chattering when there is strong disturbance in the system. A novel sliding mode controller based on extended disturbance observer is studied for a class of underactuated systems in reference, aiming to cut down the chattering effect in [28]. But small oscillation still exists in the system state when disturbance is added. Document [29] proposes an adaptive hierarchical sliding mode control method based on extended state observer for the practical application of spherical robots. The designed closed-loop control system of the spherical robot possesses robust and adaptive capabilities to overcome the uncertain rolling resistance but the response time of the system is slow and chattering exists. The proposed controller strategy that the integral sliding mode control and the optimal feedback control law is composed in [30]. The main advantages of the proposed approach are ensuring the robustness throughout the whole system response against the uncertainties, decrease the chattering effect and eliminate the reaching phase. But the simulation experiment system is linearized. A unified adaptive second order sliding mode control method is devised. By using the proposed control structure, the upper bounds of uncertainties are not required, the over-estimation of the control gains are avoided, and the chattering of the conventional sliding mode controllers can be attenuated in [31]. But only simple disturbance phenomena are analyzed, and complex disturbance factors are not analyzed.

In this paper, a hierarchical second-order fast terminal sliding mode based on disturbance observer control approach is proposed for a class of underactuated systems. The contributions of this paper are as follows.

1. The sliding mode controller are designed by using the second-order hierarchical fast terminal sliding mode surface and variable coefficient double power reaching law to reduce the chattering of the system.

2. A hyperbolic tangent nonlinear disturbance observer, which is synchronized with it, has been designed to estimate the uncertainty and external disturbance of the system.

3. The stability of sliding surface at all levels is proof. Meanwhile, the effectiveness of the method is verified by the numerical simulation experiment of inverted pendulum.

The rest of this paper is organized as follows. A class of underactuated systems is formulated in Section 2. An effective second-order hierarchical fast terminal sliding mode Controller based on disturbance observer is devised, and the stability of sliding surface at all levels is analyzed in Section 3. Section 4 conduct the simulation and present the results. Finally, some conclusions are given in Section 5.

\section{Problem Formulation}

Consider the following dynamic model of a cart-pole system as [32]:

$$
\left\{\begin{array}{l}
\dot{x}_{1}(t)=x_{2}(t) \\
\dot{x}_{2}(t)=f_{1}(\boldsymbol{x}, t)+b_{1}(\boldsymbol{x}, t) u(t)+d_{1}(t) \\
\dot{x}_{3}(t)=x_{4}(t) \\
\dot{x}_{4}(t)=f_{2}(\boldsymbol{x}, t)+b_{2}(\boldsymbol{x}, t) u(t)+d_{2}(t)
\end{array}\right.
$$

where $\boldsymbol{x}=\left[x_{1}, x_{2}, x_{3}, x_{4}\right]^{\prime}$ represent the state variable; $f_{1}(\boldsymbol{x}, t), b_{1}(\boldsymbol{x}, t), f_{2}(\boldsymbol{x}, t)$ and $b_{2}(\boldsymbol{x}, t)$ denote the nonlinear functions representing system dynamics; $u(t)$ indicates the control input; $d_{1}(t)$ and $d_{2}(t)$ are bounded external disturbances and system parameter perturbations.

The terms $f_{1}(\boldsymbol{x}, t)$ and $f_{2}(\boldsymbol{x}, t)$ can be expressed as:

$$
\left\{\begin{array}{l}
f_{1}(\boldsymbol{x}, t)=f_{1}{ }^{\prime}(\boldsymbol{x}, t)+\Delta f_{1}(\boldsymbol{x}, t) \\
f_{2}(\boldsymbol{x}, t)=f_{2}{ }^{\prime}(\boldsymbol{x}, t)+\Delta f_{2}(\boldsymbol{x}, t)
\end{array}\right.
$$

where $f_{1}{ }^{\prime}(x, t)$ and $f_{2}{ }^{\prime}(x, t)$ are the known parts of $f_{1}(x, t)$ and $f_{2}(x, t) . \Delta f_{1}(x, t)$ and $\Delta f_{2}(x, t)$ are the unknown parts of $f_{1}(x, t)$ and $f_{2}(x, t)$.

The dynamic equation of (1) can also be written as:

$$
\left\{\begin{array}{l}
\dot{x}_{1}(t)=x_{2}(t) \\
\dot{x}_{2}(t)=f_{1}{ }^{\prime}(\boldsymbol{x}, t)+b_{1}(\boldsymbol{x}, t) u(t)+n_{1}(t) \\
\dot{x}_{3}(t)=x_{4}(t) \\
\dot{x}_{4}(t)=f_{2}{ }^{\prime}(\boldsymbol{x}, t)+b_{2}(\boldsymbol{x}, t) u(t)+n_{2}(t)
\end{array}\right.
$$

where $n_{1}=\Delta f_{1}(\boldsymbol{x}, t)+d_{1}(t)$ and $n_{2}=\Delta f_{2}(\boldsymbol{x}, t)+d_{2}(t)$ are the terms of bounded external disturbances and system parameter perturbations.

Assumption 1. The system perturbations are assumed to be bounded as $n_{1} \leq \delta_{1}$ and $n_{2} \leq \delta_{2}$, where $\delta_{1}$ and $\delta_{2}$ are unknown positive constants.

\section{Main Results}

The control objective of the system is to design a robust controller that enables accurate and fast stable even in the presence of model uncertainties and external disturbances. In order to reach the target, a hierarchical second-order fast terminal sliding mode control scheme combining disturbance observer is designed.

\subsection{Disturbance Observer for Hyperbolic Tangent Nonlinear Function}

The tracking differentiator (TD) was first proposed by the researcher Han and others in China in 1994 [33]. It is used in practical engineering problems to extract continuous filtered signals and differential signals from discontinuous or random noise measurement signals.

In this paper, nonlinear tracking differentiator is constructed by hyperbolic tangent nonlinear function as follows [34]. 


$$
\left\{\begin{array}{l}
\dot{x}_{1}=x_{2} \\
\dot{x}_{2}=-R^{2}\left[m_{1} \tanh \left(h_{1}\left(x_{1}-v(t)\right)\right)+m_{2} \tanh \left(h_{2} x_{2} / R\right)\right]
\end{array}\right.
$$

where $\quad R>0, m_{1}>0, h_{1}>0, m_{2}>0, h_{2}>0 \quad$ are design parameters. The stability and convergence of system (4) are proved and detailed regulating rules of design parameters are given in [34]. A special example of TANH-TD can be obtained when $m_{1}=m_{2}, h_{1}=h_{2}$, so that it has fewer tuning parameters and will be more convenient for engineering applications.

Theorem 1. No loss of generality, we consider the underactuated mechanical system (3). The design of nonlinear disturbance observer based on tracking differentiator, described as:

$$
\left\{\begin{array}{l}
\dot{\hat{x}}_{2}=f_{1}^{\prime}(\boldsymbol{x})+b_{1}(\boldsymbol{x}) u+\hat{n}_{1} \\
\dot{\hat{n}}_{1}=-R^{2}\left[m_{1} \tanh \left(h_{1}\left(\hat{x}_{2}-x_{2}\right)\right)+m_{2} \tanh \left(h_{2} \hat{n}_{1} / R\right)\right] \\
\dot{\hat{x}}_{4}=f_{2}^{\prime}(\boldsymbol{x})+b_{2}(\boldsymbol{x}) u+\hat{n}_{2} \\
\dot{\hat{n}}_{2}=-R^{2}\left[m_{1} \tanh \left(h_{1}\left(\hat{x}_{4}-x_{4}\right)\right)+m_{2} \tanh \left(h_{2} \hat{n}_{2} / R\right)\right]
\end{array}\right.
$$

where $\hat{x}_{2}, \hat{n}_{1}, \hat{x}_{4}, \hat{n}_{2}$ are the estimates of $x_{2}, n_{1}, x_{4}, n_{2}$, respectively. If $T>0, R>0$, we get:

$$
\left\{\begin{array}{l}
\lim _{R \rightarrow \infty} \int_{0}^{T}\left|\hat{x}_{2}-x_{2}\right| d t=0 \\
\lim _{R \rightarrow \infty} \int_{0}^{T}\left|\hat{x}_{4}-x_{4}\right| d t=0
\end{array}\right.
$$

In other words, $\hat{x}_{2} \rightarrow x_{2}, \hat{x}_{4} \rightarrow x_{4}$. In addition, we have $\hat{n}_{1} \rightarrow n_{1}, \hat{n}_{2} \rightarrow n_{2}$ according to the equation of system (3) and (5).

Proof: When $R \rightarrow \infty$, the following equation can be obtained:

$$
\left\{\begin{array}{l}
\left|\dot{\hat{n}}_{1}\right|=\left|-R^{2}\left[m_{1} \tanh \left(h_{1}\left(\hat{x}_{2}-x_{2}\right)\right)+m_{2} \tanh \left(h_{2} \hat{n}_{1} / R\right)\right]\right| \\
\left|\dot{\hat{n}}_{2}\right|=\mid-R^{2}\left[m_{1} \tanh \left(h_{1}\left(\hat{x}_{4}-x_{4}\right)\right)+m_{2} \tanh \left(h_{2} \hat{n}_{2} / R\right)\right]
\end{array}\right.
$$

That is, the varies in $\hat{n}_{1}$ and $\hat{n}_{2}$ is much faster than $f_{1}^{\prime}(\boldsymbol{x})+b_{1}(\boldsymbol{x}) u$ and $f_{2}^{\prime}(\boldsymbol{x})+b_{2}(\boldsymbol{x}) u$, respectively.

Meanwhile, we can clearly get the equation as:

$$
\left\{\begin{array}{l}
\lim _{R \rightarrow \infty} d\left(f_{1}^{\prime}(\boldsymbol{x})+b_{1}(\boldsymbol{x}) u+\hat{n}_{1}\right) / d t=\dot{\hat{n}}_{1} \\
\lim _{R \rightarrow \infty} f_{1}^{\prime}(\boldsymbol{x})+b_{1}(\boldsymbol{x}) u+\hat{n}_{1} / R=\hat{n}_{1} / R \\
\lim _{R \rightarrow \infty} d\left(f_{2}^{\prime}(\boldsymbol{x})+b_{2}(\boldsymbol{x}) u+\hat{n}_{2}\right) / d t=\dot{\hat{n}}_{2} \\
\lim _{R \rightarrow \infty} f_{2}^{\prime}(\boldsymbol{x})+b_{2}(\boldsymbol{x}) u+\hat{n}_{2} / R=\hat{n}_{2} / R
\end{array}\right.
$$

Therefore, when we regard " $f_{1}^{\prime}(\boldsymbol{x})+b_{1}(\boldsymbol{x}) u+\hat{n}_{1}$ " as " $x_{2}$ " and $" f_{2}^{\prime}(\boldsymbol{x})+b_{2}(\boldsymbol{x}) u+\hat{n}_{2} "$ as " $x_{4} "$, it is clear that (5), (6) are established according to theorem of [34]. This is the proof of completion.

Remark 1. Please note that in the actual system, any control input is limited. In other words, bandwidth and speed are bounded for any control input $u$. Therefore, it is reasonable to assume that the varies in $\hat{n}_{1}$ and $\hat{n}_{2}$ is much faster than $f_{1}^{\prime}(\boldsymbol{x})+b_{1}(\boldsymbol{x}) u$ and $f_{2}^{\prime}(\boldsymbol{x})+b_{2}(\boldsymbol{x}) u$.

\subsection{Second-Order Hierarchical Fast Terminal Sliding Mode}

Corresponding to the two groups of the state variables $\left(x_{1}, x_{2}\right)$ and $\left(x_{3}, x_{4}\right)$ of two subsystems we construct a pair of suitable integral sliding surfaces as:

$$
\left\{\begin{array}{l}
s_{1}=x_{1}+\lambda_{1} \int x_{1} d t \\
\dot{s}_{1}=x_{2}+\lambda_{1} x_{1} \\
s_{2}=x_{3}+\lambda_{2} \int x_{3} d t \\
\dot{s}_{1}=x_{4}+\lambda_{2} x_{3}
\end{array}\right.
$$

where $\lambda_{1}$ and $\lambda_{2}$ are positive constants.

According to (9), the following first- level sliding surface is defined

$$
\left\{\begin{array}{l}
S_{\text {sosm } 1}=\alpha_{1} s_{1}+\beta_{1} s_{1}{ }^{q_{1} / p_{1}}+\dot{s}_{1} \\
S_{\text {sosm } 2}=\alpha_{2} s_{2}+\beta_{2} s_{2}{ }^{q_{2} / p_{2}}+\dot{s}_{2}
\end{array}\right.
$$

where $\alpha_{1}, \beta_{1}, \alpha_{2}, \beta_{2}$ are positive constants. $q_{1}, p_{1}, q_{2}, p_{2}$ are all positive odd numbers and $q_{1}<p_{1}, q_{2}<p_{2}$. Using the equivalent control method the equivalent control law of the subsystems can be obtained as:

$$
\begin{aligned}
& u_{e q 1}=-\frac{f_{1}{ }^{\prime}(\boldsymbol{x}, t)+\hat{n}_{1}+\lambda_{1} x_{2}+\dot{s}_{1}\left(\alpha_{1}+\beta_{1} \frac{q_{1}}{p_{1}} s_{1}^{q_{1} / p_{1}-1}\right)}{b_{1}(\boldsymbol{x}, t)} \\
& u_{e q 2}=-\frac{f_{2}{ }^{\prime}(\boldsymbol{x}, t)+\hat{n}_{2}+\lambda_{2} x_{4}+\dot{s}_{2}\left(\alpha_{2}+\beta_{2} \frac{q_{2}}{p_{2}} s_{2}^{q_{2} / p_{2}-1}\right)}{b_{2}(\boldsymbol{x}, t)}
\end{aligned}
$$

The under-actuated system has the characteristic of controlling the multiple outputs with less input. Therefore, the total control input includes the equivalent inputs for all subsystems. We define the total control as:

$$
u=u_{e q 1}+u_{e q 2}+u_{s w}
$$

where $u_{s w}$ is the switch control part of the sliding controller. According to the first- level sliding surface of all subsystem, defining the second-level sliding surface and double power approaching law with the variable coefficient as:

$$
S=a_{1} S_{\text {sosm } 1}+a_{2} S_{\text {sosm } 2}
$$




$$
\dot{S}=\left(-k_{1}|S|^{c_{1}} \operatorname{sgn}(S)-k_{2}|S|^{c_{2}} \operatorname{sgn}(S)\right) \cdot\left(1+2\|\boldsymbol{M}\|_{\infty}\right)
$$

where $\boldsymbol{M}=\left[S_{\text {sosm } 1}, S_{\text {sosm } 2}\right] . a_{1}, a_{2}$ are sliding-mode parameters which maybe remain constant or change according to different conditions. $k_{1}, k_{2}, c_{1}, c_{2}$ are positive constants and $0<c_{1}<1, c_{2}>1$.

According to the Lyapunov stability theorem, we derive the switching control law. The positive definite Lyapunov energy function can be defined as follows.

$$
V(t)=\frac{1}{2} S^{2}
$$

Differentiating $V(t)$ with respect to time t obtains

$$
\begin{aligned}
\dot{V} & =S \dot{S}=S\left(a_{1} \dot{S}_{s o s m 1}+a_{2} \dot{S}_{s o s m 2}\right) \\
& =S\left[\begin{array}{l}
a_{1}\left(f_{1}^{\prime}(\boldsymbol{x}, t)+b_{1}(\boldsymbol{x}, t)\left(u_{e q 1}+u_{e q 2}+u_{s w}\right)+n_{1}\right. \\
\left.+\lambda_{1} x_{2}+\dot{s}_{1}\left(\alpha_{1}+\beta_{1} \frac{q_{1}}{p_{1}} s_{1}^{q_{1} / p_{1}-1}\right)\right)+a_{2}\left(f_{2}{ }^{\prime}(\boldsymbol{x}, t)\right. \\
+b_{2}(\boldsymbol{x}, t)\left(u_{e q 1}+u_{e q 2}+u_{s w}\right)+n_{2}+\lambda_{2} x_{4}+\dot{s}_{2} \\
\left.\left(\alpha_{2}+\beta_{2} \frac{q_{2}}{p_{2}} s_{2}^{q_{2} / p_{2}-1}\right)\right)
\end{array}\right] \\
& =S\left[\begin{array}{l}
a_{1}\left(n_{1}-\hat{n}_{1}+b_{1}(\boldsymbol{x}, t)\left(u_{e q 2}+u_{s w}\right)\right)+a_{2}\left(n_{2}-\hat{n}_{2}\right. \\
\left.+b_{2}(\boldsymbol{x}, t)\left(u_{e q 1}+u_{s w}\right)\right)
\end{array}\right] \\
& =S\left[\begin{array}{l}
a_{1}\left(n_{1}-\hat{n}_{1}+b_{1}(\boldsymbol{x}, t) u_{e q 2}\right)+a_{1} b_{1}(\boldsymbol{x}, t) u_{s w} \\
+a_{2}\left(n_{2}-\hat{n}_{2}+b_{2}(\boldsymbol{x}, t) u_{e q 1}\right)+a_{2} b_{2}(\boldsymbol{x}, t) u_{s w}
\end{array}\right] \\
& =S\left[\begin{array}{l}
a_{1}\left(n_{1}-\hat{n}_{1}+b_{1}(\boldsymbol{x}, t) u_{e q 2}\right)+a_{2}\left(n_{2}-\hat{n}_{2}\right. \\
\left.+b_{2}(\boldsymbol{x}, t) u_{e q 1}\right)+\left(a_{1} b_{1}(\boldsymbol{x}, t)+a_{2} b_{2}(\boldsymbol{x}, t)\right) u_{s w}
\end{array}\right]
\end{aligned}
$$

The switching control law is defined as:

$$
u_{s w}=\frac{\left[\begin{array}{l}
a_{1} b_{1}(\boldsymbol{x}, t) u_{e q 2}+a_{2} b_{2}(\boldsymbol{x}, t) u_{e q 1}+\left(k_{1}|S|^{c_{1}} \operatorname{sgn}(S)\right. \\
\left.+k_{2}|S|^{c_{2}} \operatorname{sgn}(S)\right)\left(1+2\|\boldsymbol{M}\|_{\infty}\right)
\end{array}\right]}{-\left(a_{1} b_{1}(\boldsymbol{x}, t)+a_{2} b_{2}(\boldsymbol{x}, t)\right)}
$$

Therefore, the general control law of the system is given as follows.

$$
\begin{aligned}
& u=u_{e q 1}+u_{e q 2}+u_{s w} \\
& =\frac{\left[\begin{array}{l}
a_{1} b_{1}(\boldsymbol{x}, t) u_{e q 1}+a_{2} b_{2}(\boldsymbol{x}, t) u_{e q 2}-\left(k_{1}|S|^{c_{1}} \operatorname{sgn}(S)\right. \\
\left.+k_{2}|S|^{c_{2}} \operatorname{sgn}(S)\right)\left(1+2\|\boldsymbol{M}\|_{\infty}\right)
\end{array}\right]}{\left(a_{1} b_{1}(\boldsymbol{x}, t)+a_{2} b_{2}(\boldsymbol{x}, t)\right)}
\end{aligned}
$$

\subsection{Stability Analysis}

Lemma 1. [35]: Set $\boldsymbol{x} \in M \subset R^{n}, \dot{\boldsymbol{x}}=f(\boldsymbol{x}), f: R^{n} \rightarrow R$ as the continuous functions defined in the equilibrium point region $M$. Assuming that a continuous function $V: M \rightarrow R$ satisfies the following conditions:

1) $V$ is positive definite.

2) $\dot{V}$ is negative definite except for the equilibrium point.
3) Real number $k>0, \alpha>0$ and region $N \subset M$ make $\dot{V}+k V^{\alpha} \leq 0$, the function $\dot{\boldsymbol{x}}=f(\boldsymbol{x})$ converge sat balanced zero point with infinite time.

Theorem 2. The under-actuated system (3) is adopted in the control law of (19), and the second-level sliding surface $S$ and $\dot{S}$ converge to the following regions in a finite time.

$$
\begin{aligned}
|S| \leq \min \left(\left(\frac{D_{M}}{k_{1}^{*}}\right)^{1 / c_{1}},\left(\frac{D_{M}}{k_{2}{ }^{*}}\right)^{1 / c_{2}}\right) \\
|\dot{S}| \leq \min \left(D_{M}\left(1+2\|\boldsymbol{M}\|_{\infty}\right), k_{1}^{*}\left(\frac{D_{M}}{k_{2}}\right)^{c_{1} / c_{2}}\right) \\
+\min \left(k_{2}^{*}\left(\frac{D_{M}}{k_{1}}\right)^{c_{2} / c_{1}}, D_{M}\left(1+2\|\boldsymbol{M}\|_{\infty}\right)\right)
\end{aligned}
$$

Proof: Substitute (18) into (17) to obtain:

$$
\begin{aligned}
\dot{V} & =S\left[\begin{array}{l}
a_{1}\left(n_{1}-\hat{n}_{1}\right)+a_{2}\left(n_{2}-\hat{n}_{2}\right)-\left(k_{1}|S|^{c_{1}} \operatorname{sgn}(S)\right. \\
\left.+k_{2}|S|^{c_{2}} \operatorname{sgn}(S)\right) \cdot\left(1+2\|\boldsymbol{M}\|_{\infty}\right)
\end{array}\right] \\
& =S\left[a_{1}\left(n_{1}-\hat{n}_{1}\right)+a_{2}\left(n_{2}-\hat{n}_{2}\right)\right]-k_{1}^{*}|S|^{c_{1}+1}-k_{2}^{*}|S|^{c_{2}+1} \\
& \leq D_{M}|S|-k_{1}^{*}|S|^{c_{1}+1}-k_{2}^{*}|S|^{c_{2}+1}
\end{aligned}
$$

where $\quad D_{M}=\sup _{t \geq 0}\left|\left[a_{1}\left(n_{1}-\hat{n}_{1}\right)+a_{2}\left(n_{2}-\hat{n}_{2}\right)\right]\right| \quad, \quad k_{1}^{*}=k_{1}\left(1+2\|\boldsymbol{M}\|_{\infty}\right)$, $k_{2}^{*}=k_{2}\left(1+2\|\boldsymbol{M}\|_{\infty}\right)$. Then it becomes

$$
\begin{aligned}
& \dot{V} \leq-k_{1}{ }^{*}|S|^{c_{1}+1}-\left(k_{2}{ }^{*}|S|^{c_{2}}-D_{M}\right)|S| \\
& \dot{V} \leq-k_{2}{ }^{*}|S|^{c_{2}+1}-\left(k_{1}^{*}|S|^{c_{1}}-D_{M}\right)|S|
\end{aligned}
$$

If $k_{2}{ }^{*}|S|^{c_{2}} \geq D_{M}$ is satisfied, then

$$
\dot{V} \leq-k_{1}^{*}|S|^{c_{1}+1}=-k_{1}^{*} V^{\left(c_{1}+1\right) / 2}
$$

Lemma 1 shows that the system converges with respect to equilibrium zeros in finite time. Since $k_{2}{ }^{*}=k_{2}\left(1+2\|\boldsymbol{M}\|_{\infty}\right) \geq k_{2}$, we can get

$$
|S| \leq\left(\frac{D_{M}}{k_{2}{ }^{*}}\right)^{1 / c_{2}} \leq\left(\frac{D_{M}}{k_{2}}\right)^{1 / c_{2}}
$$

outside the area $\dot{V} \leq 0$. Therefore, the convergence region at this time is (26).

If $k_{1}^{*}|S|^{c_{1}} \geq D_{M}$ is satisfied, then

$$
\dot{V} \leq-k_{2}^{*}|S|^{c_{2}+1}=-k_{2}^{*} V^{\left(c_{2}+1\right) / 2}
$$

Lemma 1 shows that the system converges with respect to 
equilibrium zeros in finite time. Since $k_{1}^{*}=k_{1}\left(1+2\|\boldsymbol{M}\|_{\infty}\right) \geq k_{1}$, we can get

$$
|S| \leq\left(\frac{D_{M}}{k_{1}^{*}}\right)^{1 / c_{1}} \leq\left(\frac{D_{M}}{k_{1}}\right)^{1 / c_{1}}
$$

outside the area $\dot{V} \leq 0$. Therefore, the convergence region at this time is (28).

By synthesizing (26) and (28), $S$ can converges to the following regions in finite time.

$$
|S| \leq \min \left(\left(\frac{D_{M}}{k_{1}}\right)^{1 / c_{1}},\left(\frac{D_{M}}{k_{2}}\right)^{1 / c_{2}}\right)
$$

Substituting the previous equation into (15), we can get:

$$
\begin{aligned}
|\dot{S}| & \leq k_{1}^{*}|S|^{c_{1}} \operatorname{sgn}(S)+k_{2}^{*}|S|^{c_{2}} \operatorname{sgn}(S) \\
& \leq k_{1}^{*}|S|^{c_{1}}+k_{2}^{*}|S|^{c_{2}} \\
& \leq k_{1}^{*} \cdot \min \left(\left(\frac{D_{M}}{k_{1}}\right)^{1 / c_{1}},\left(\frac{D_{M}}{k_{2}}\right)^{1 / c_{2}}\right)^{c_{1}} \\
& +k_{2}^{*} \cdot \min \left(\left(\frac{D_{M}}{k_{1}}\right)^{1 / c_{1}},\left(\frac{D_{M}}{k_{2}}\right)^{1 / c_{2}}\right)^{c_{2}} \\
& =\min \left(D_{M}\left(1+2\|\boldsymbol{M}\|_{\infty}\right), k_{1}^{*}\left(\frac{D_{M}}{k_{2}}\right)^{c_{1} / c_{2}}\right) \\
& +\min \left(k_{2}^{*}\left(\frac{D_{M}}{k_{1}}\right)^{c_{2} / c_{1}}, D_{M}\left(1+2\|\boldsymbol{M}\|_{\infty}\right)\right)
\end{aligned}
$$

In conclusion, the theorem 2 is proved to be correct.

Theorem 3. If the control law of (19) is used for the underactuated system (3) and the sliding surface is designed as (10), (14). The first-layer sliding mode surfaces $S_{\text {sosm } 1}, S_{\text {sosm } 2}$ are also asymptotically stable.

Proof: According to the theorem 2, we can have $|S| \in L_{\infty}$ and $S^{2} \in L_{\infty}$. By analyzing the first sliding mode surfaces, we can easily get that $s_{1} \in L_{\infty}, s_{2} \in L_{\infty}, \dot{s}_{1} \in L_{\infty}, \dot{s}_{2} \in L_{\infty}$.

Furthermore, we have

$$
\begin{aligned}
\int_{0}^{\infty} S^{2} d \tau & =\int_{0}^{\infty}\left(a_{1} S_{\text {sosm } 1}+a_{2} S_{\text {sosm } 2}\right)^{2} d \tau \\
= & \int_{0}^{\infty}\left(\begin{array}{l}
a_{1}^{2} S_{\text {sosm } 1}^{2}+2 a_{1} a_{2} S_{\text {sosm } 1} S_{\text {sosm } 2} \\
+a_{2}^{2} S_{\text {sosm } 2}^{2}
\end{array}\right)^{2} d \tau
\end{aligned}
$$

Owing to $\int_{0}^{\infty} S^{2} d \tau<\infty$ and $2 a_{1} a_{2} S_{\text {sosm } 1} S_{\text {sosm } 2} \leq a_{1}{ }^{2} S_{\text {sosm } 1}{ }^{2}+a_{2}{ }^{2} S_{\text {sosm } 2}{ }^{2}$, the following inequality is satisfied.

$$
\int_{0}^{\infty} 4 a_{1} a_{2} S_{\text {sosm } 1} S_{\text {sosm } 2} d \tau \leq \int_{0}^{\infty}\left(a_{1} S_{\text {sosm } 1}+a_{2} S_{\text {sosm } 2}\right)^{2} d \tau<\infty
$$

According to (31) and (32), we can have

$$
\int_{0}^{\infty} S_{\text {sosm } 1}^{2} d \tau<\infty, \int_{0}^{\infty} S_{\text {sosm } 2}{ }^{2} d \tau<\infty
$$

Therefore, $s_{1} \in L_{\infty}, s_{2} \in L_{\infty}, \dot{s}_{1} \in L_{\infty}, \dot{s}_{2} \in L_{\infty}$, and it can be easily obtained that $\lim _{t \rightarrow \infty} S_{\text {sosm } 1}=0$ in terms of the Barbalat's lemma. Same argument, it can be attested that $\lim _{t \rightarrow \infty} S_{\text {sosm } 2}=0$. In summary, the Theorem 3 is proved to be correct.

\section{Simulation Results}

In order to validate the suggested control technique, a car inverted pendulum system is simulated some comparisons between the suggested technique and the methods of $[36,24]$ are presented.

The schematic diagram of the inverted pendulum system is shown in Figure 1. The dynamic equation of the cart-pole system can be exhibited in the form of (3) with the functions $f_{1}{ }^{\prime}(x, t), b_{1}(x, t), f_{2}{ }^{\prime}(x, t)$ and $b_{2}(x, t)$ as:

$$
\left\{\begin{array}{l}
f_{1}{ }^{\prime}(\boldsymbol{x}, t)=\frac{m_{t} g \sin \left(x_{1}\right)-m_{p} L \sin \left(x_{1}\right) \cos \left(x_{1}\right) x_{2}^{2}}{L\left(\left(\frac{4}{3}\right) m_{t}-m_{p} \cos ^{2}\left(x_{1}\right)\right)} \\
b_{1}(\boldsymbol{x}, t)=\frac{\cos \left(x_{1}\right)}{L\left(\left(\frac{4}{3}\right) m_{t}-m_{p} \cos ^{2}\left(x_{1}\right)\right)}, \\
f_{2}{ }^{\prime}(\boldsymbol{x}, t)=\frac{-\left(\frac{4}{3}\right) m_{p} L x_{2}^{2} \sin \left(x_{1}\right)+m_{p} g \sin \left(x_{1}\right) \cos \left(x_{1}\right)}{\left(\frac{4}{3}\right) m_{t}-m_{p} \cos ^{2}\left(x_{1}\right)} \\
b_{2}(\boldsymbol{x}, t)=\frac{4}{3\left(\left(\frac{4}{3}\right) m_{t}-m_{p} \cos ^{2}\left(x_{1}\right)\right)}
\end{array}\right.
$$

where $m_{t}$ is the total mass of the cart-pole system which contains the quality of the pole $\left(m_{p}\right)$ and the mass of the cart $\left(m_{c}\right), x_{1}$ represents the swing angle of the pole, $x_{2}$ expresses the swing speed of the pole, $x_{3}$ denotes the position of the cart, $x_{4}$ indicates the cart velocity. Contrasting control schemes are considered as below:

(a) The sliding surface, reaching law and controller of hierarchical sliding mode control method (HSM) based on exponential reaching law are as follows [30]:

$$
\begin{gathered}
s_{1}=\alpha_{1} x_{1}+x_{2}, s_{2}=\alpha_{2} x_{3}+x_{4}, S=a_{1} s_{1}+a_{2} s_{2}, \\
\dot{S}=-\rho S-w \operatorname{sgn}(S), u=u_{e q 1}+u_{e q 2}+u_{s w}, \\
u_{e q 1}=-\frac{f_{1}(\boldsymbol{x})+\alpha_{1} x_{2}}{b_{1}(\boldsymbol{x})}, u_{e q 2}=-\frac{f_{2}(\boldsymbol{x})+\alpha_{2} x_{4}}{b_{2}(\boldsymbol{x})}, \\
u_{s w}=\frac{a_{1} b_{1} u_{e q 2}+a_{2} b_{2} u_{e q 1}+\rho S+w \operatorname{sgn}(S)}{-\left(a_{1} b_{1}+a_{2} b_{2}\right)} .
\end{gathered}
$$

(b) The sliding surface, reaching law and controller of hierarchical fast terminal sliding mode control method (HFTSM) based on exponential reaching law are as follows [21]: 


$$
\begin{aligned}
s_{1} & =\alpha_{1} x_{1}+\beta_{1} x_{1} q_{1} / p_{1}+x_{2}, s_{2}=\alpha_{2} x_{3}+\beta_{2} x_{3}^{q_{2} / p_{2}}+x_{4}, \\
S & =a_{1} s_{1}+a_{2} s_{2}, \dot{S}=-\rho S-w \operatorname{sgn}(S), \\
u & =u_{e q 1}+u_{e q 2}+u_{s w}, \\
u_{e q 1} & =\frac{f_{1}(\boldsymbol{x}, t)+x_{2}\left(\alpha_{1}+\beta_{1} \frac{q_{1}}{p_{1}} x_{1}^{q_{1} / p_{1}-1}\right)}{-b_{1}(\boldsymbol{x}, t)}, \\
u_{e q 2} & =-\frac{f_{2}(\boldsymbol{x}, t)+x_{4}\left(\alpha_{2}+\beta_{2} \frac{q_{2}}{p_{2}} x_{3}^{q_{2} / p_{2}-1}\right)}{b_{2}(\boldsymbol{x}, t)}, \\
u_{s w} & =\frac{\left[\begin{array}{l}
a_{1} b_{1}(\boldsymbol{x}, t) u_{e q 2}+a_{2} b_{2}(\boldsymbol{x}, t) u_{e q 1}+\rho S \\
+w \operatorname{sgn}(S)
\end{array}\right]}{-\left(a_{1} b_{1}(\boldsymbol{x}, t)+a_{2} b_{2}(\boldsymbol{x}, t)\right)} .
\end{aligned}
$$

For the simulation implementation, the constant parameters and initial conditions of the cart-pole system are set as $m_{c}=1 \mathrm{~kg}, m_{p}=0.05 \mathrm{~kg}, L=0.5 \mathrm{~m}, g=9.8 \mathrm{~m} / \mathrm{s}^{2}$, $x(0)=[-30 / 57.3,0,0,0]$. The disturbances and uncertainties terms are given as $d_{1}(t)=d_{2}(t)=0.0873 \sin (t)$, $\Delta f_{1}(\boldsymbol{x}, t)=0.1 \sin \left(x_{1}(t)\right)$ and $\Delta f_{2}(\boldsymbol{x}, t)=0.1 \sin \left(x_{3}(t)\right)$. The parameters of controllers are provided in Table 1 .

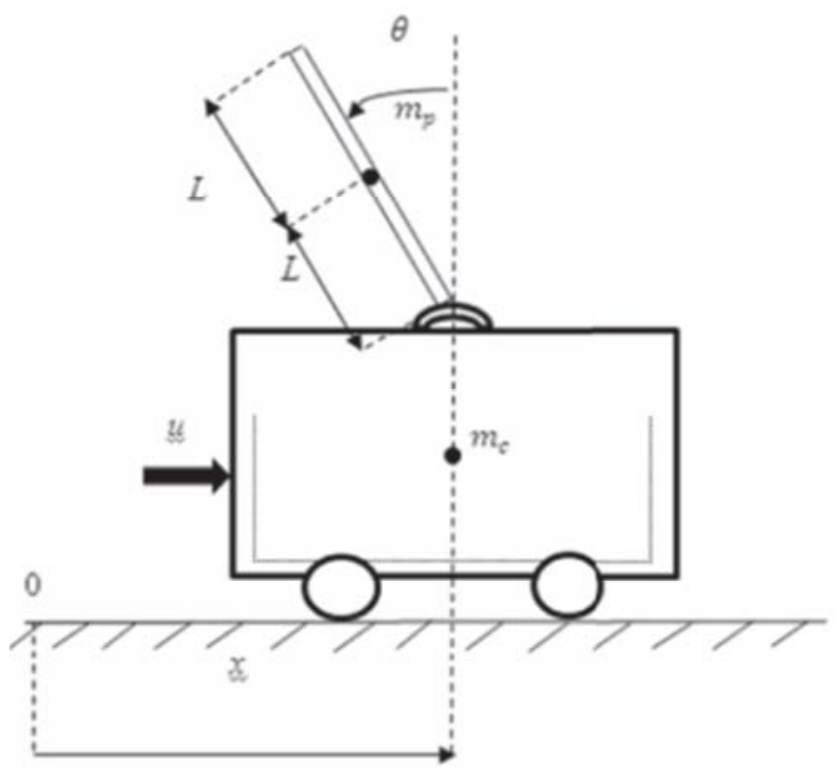

Figure 1. Inverted pendulum system.

In order to better analyze the stability of the system, the following performance evaluation indicators are given:

(1) Integral of error squared value (ISE)

$$
\mathrm{ISE}=\int_{0}^{t} x_{i}^{2}(\tau) d \tau
$$

(2) Integral of time-multiplied absolute value of error (ITAE)

$$
\mathrm{ITAE}=\int_{0}^{t} \tau\left|x_{i}(\tau)\right| d \tau
$$

The performance index values of the inverted pendulum

\begin{tabular}{|c|c|c|c|}
\hline Parameters & HSM & HFTSM & DOSHFTSM \\
\hline$\beta_{1}$ & - & 0.01 & 0.01 \\
\hline$\beta_{2}$ & - & 1 & 1 \\
\hline$\alpha_{1}$ & 3.5 & 2.7 & 3.5 \\
\hline$\alpha_{2}$ & 1.5 & 0.01 & 0.01 \\
\hline$q_{1} / p_{1}$ & - & $19 / 21$ & $19 / 21$ \\
\hline$q_{2} / p_{2}$ & - & $19 / 21$ & $19 / 21$ \\
\hline$\rho$ & 1 & 1 & - \\
\hline$w$ & 0.8 & 0.8 & - \\
\hline$a_{1}$ & -1 & -1 & -1 \\
\hline$a_{2}$ & 1 & 1 & 1 \\
\hline$\lambda_{1}$ & - & - & 0.001 \\
\hline$\lambda_{2}$ & - & - & 0.001 \\
\hline$k_{1}$ & - & - & 0.8 \\
\hline$k_{2}$ & - & - & 1 \\
\hline$c_{1}$ & - & - & 0.5 \\
\hline$c_{2}$ & - & - & 1.5 \\
\hline$m_{1}=m_{2}$ & - & - & 1 \\
\hline$h_{1}=h_{2}$ & - & - & 5 \\
\hline$R$ & - & - & 25 \\
\hline
\end{tabular}
system for the above two evaluation methods are shown in Table 2. It can be seen from Table 2 that the performance evaluation index value of the state $x_{1}$ and $x_{3}$ of the control method used in this paper are smaller than other control methods. Therefore, the system stability of DOSHFTSM is better than that of other control methods.

Table 1. Controller parameters.

Table 2. Performance indicators of inverted pendulum system.

\begin{tabular}{lllll}
\hline & ISE & \multicolumn{3}{c}{ ITAE } \\
\cline { 2 - 5 } & $\boldsymbol{x}_{1}$ & $\boldsymbol{x}_{3}$ & $\boldsymbol{x}_{1}$ & $\boldsymbol{x}_{3}$ \\
\hline HSM & 0.0657 & 0.4754 & 0.0032 & 0.0080 \\
HFTSM & 0.0611 & 0.4525 & 0.0029 & 0.0085 \\
DOSHFTSM & 0.0406 & 0.2686 & 0.0022 & 0.0063 \\
\hline
\end{tabular}

Figures 2-4 shows the response curves of the system state, the sliding surfaces, and the system phase trajectory, respectively. It can be seen from them that the system state can converge to the equilibrium point at a faster speed under the control of the proposed scheme and can intuitively show that the control process of the proposed method is smooth and jitter-free. Figure 5 show that the control method of the present invention controls the input signal to be smooth, which can reduce the wear of the motor. Figure 6 show that the disturbance observer designed in this paper can estimate the interference value very well.

In what follows, for the robustness analysis, the simulation studies are repeated with different values of the initial conditions, disturbances and uncertainties terms. The new condition is specified as $x(0)=[-45 / 57.3,0,0,0]$, $d_{1}(t)=d_{2}(t)=0.1 \sin (t), \Delta f_{1}(\boldsymbol{x}, t)=0.1 \cos \left(x_{1}(t)\right), \Delta f_{2}(\boldsymbol{x}, t)=0.5 \sin \left(x_{3}(t)\right)$. 


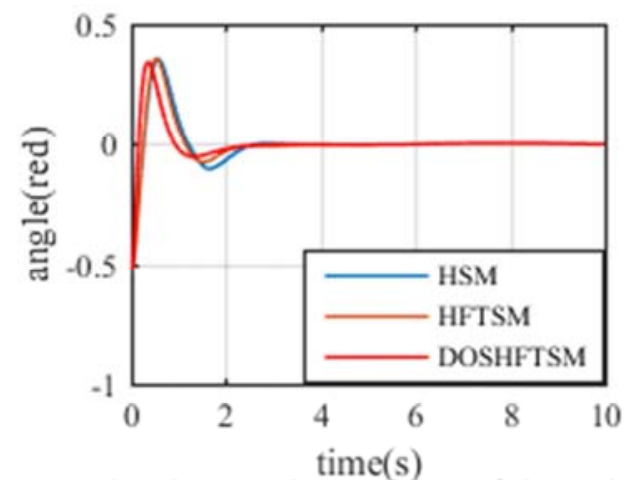

a). The angular position of the pole.

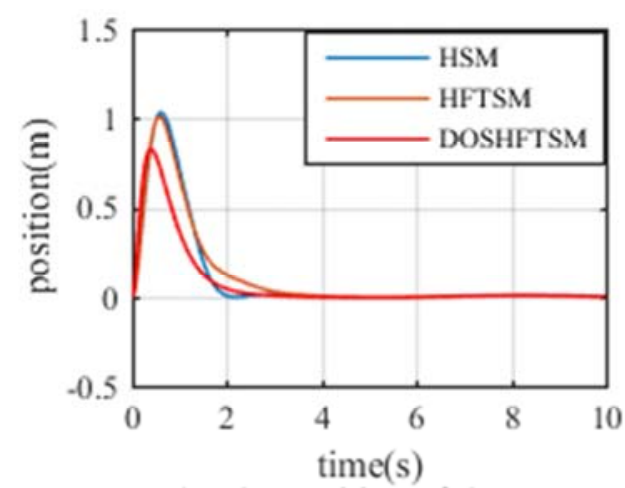

c). The position of the cart.

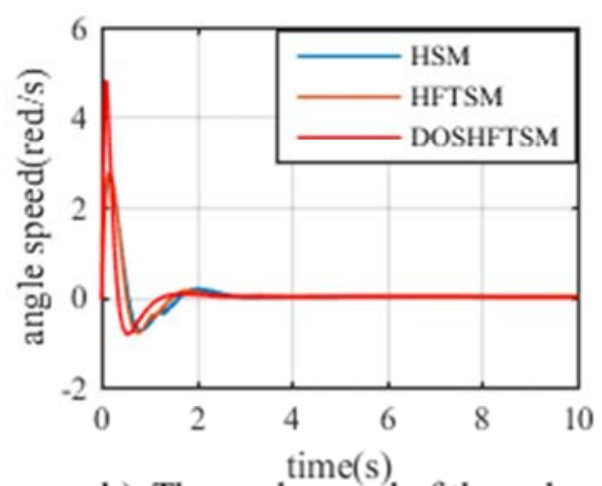

b). The angle speed of the pole.

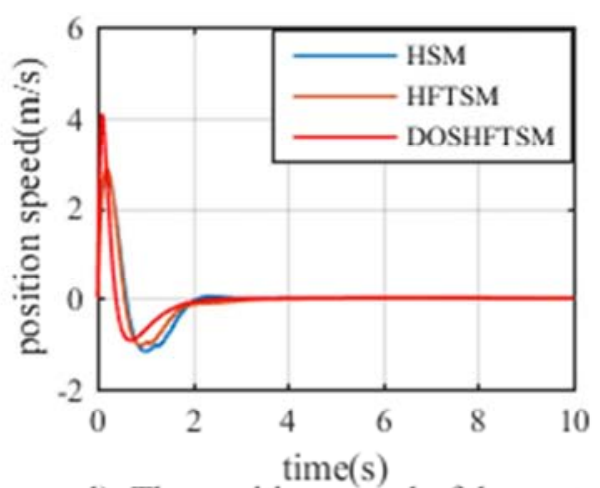

d). The position speed of the cart.

Figure 2. The response curves of the system state.

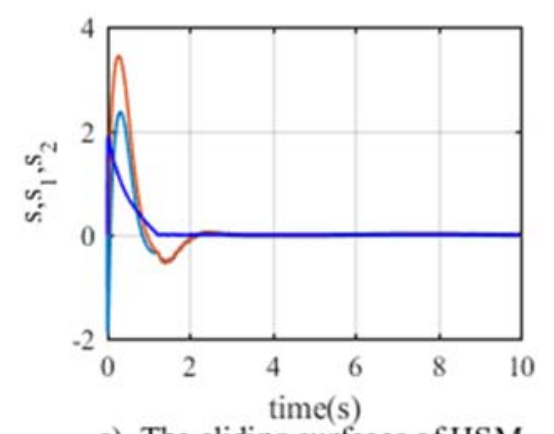

a). The sliding surfaces of HSM.

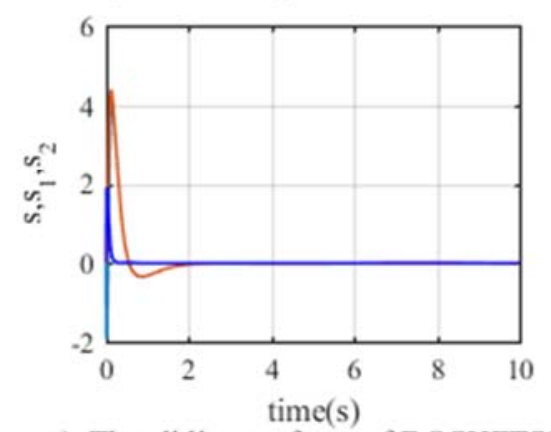

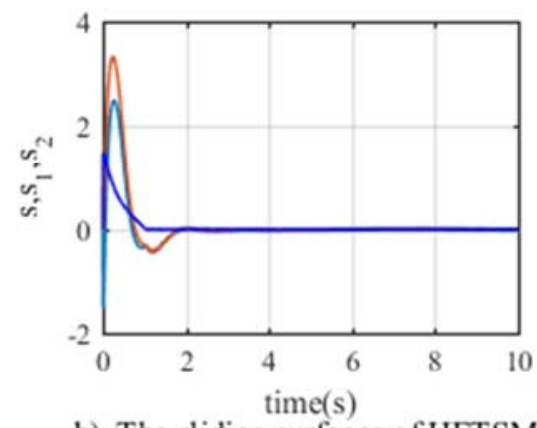

b). The sliding surfaces of HFTSM.

c). The sliding surfaces of DOSHFTSM.

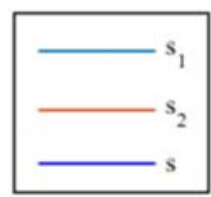

Figure 3. The response curves of the sliding surfaces.

At the same time, $5 \sin (t)$ interference signal is added to the simulation to $5 \mathrm{~s}$. Time responses of the system state, the sliding surfaces, system phase trajectory and control input signal are displayed in Figures 7-10, respectively. These simulation results also confirm that the control scheme has good robustness under different conditions. Therefore, the systems can be stably controlled with different values of the initial conditions, disturbances and uncertainties terms, too. 

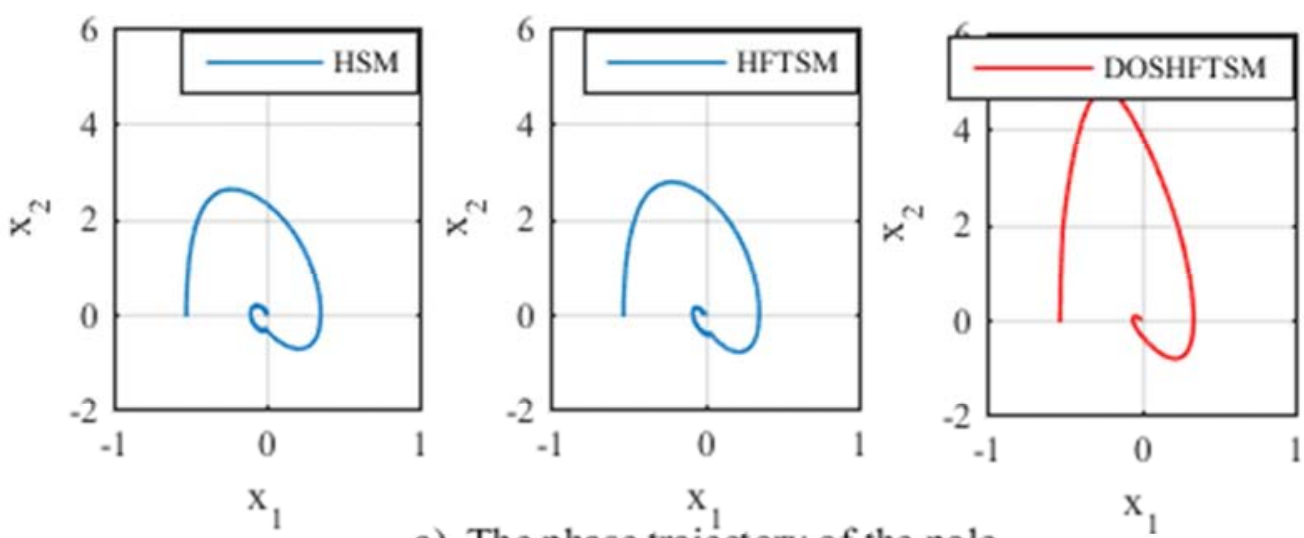

a). The phase trajectory of the pole
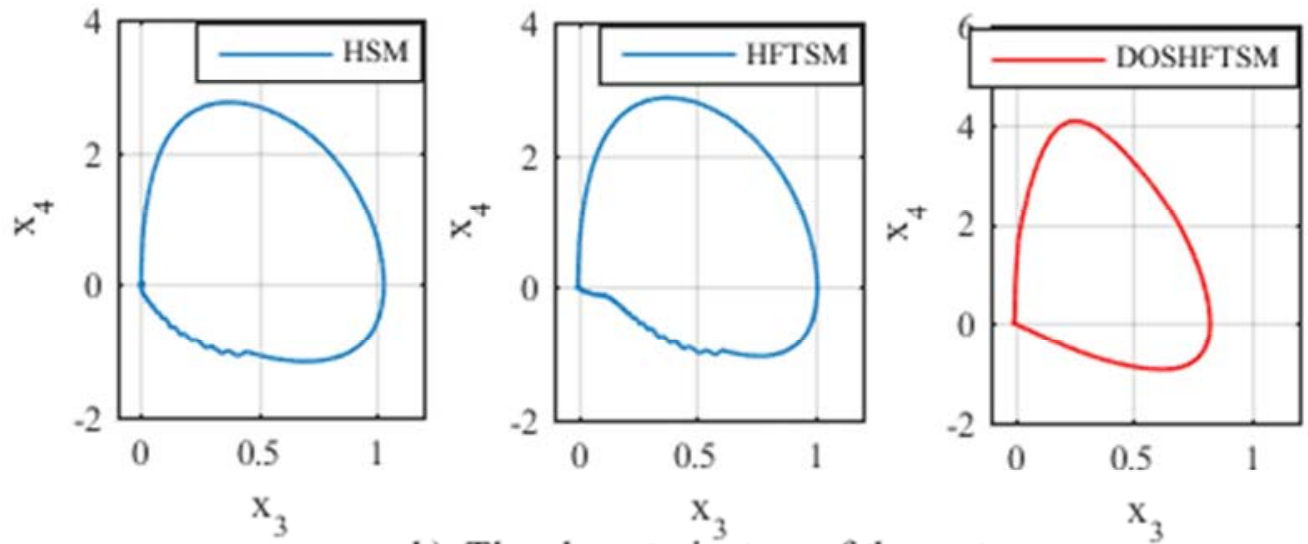

b). The phase trajectory of the cart.

Figure 4. The response curves of the system phase trajectory.

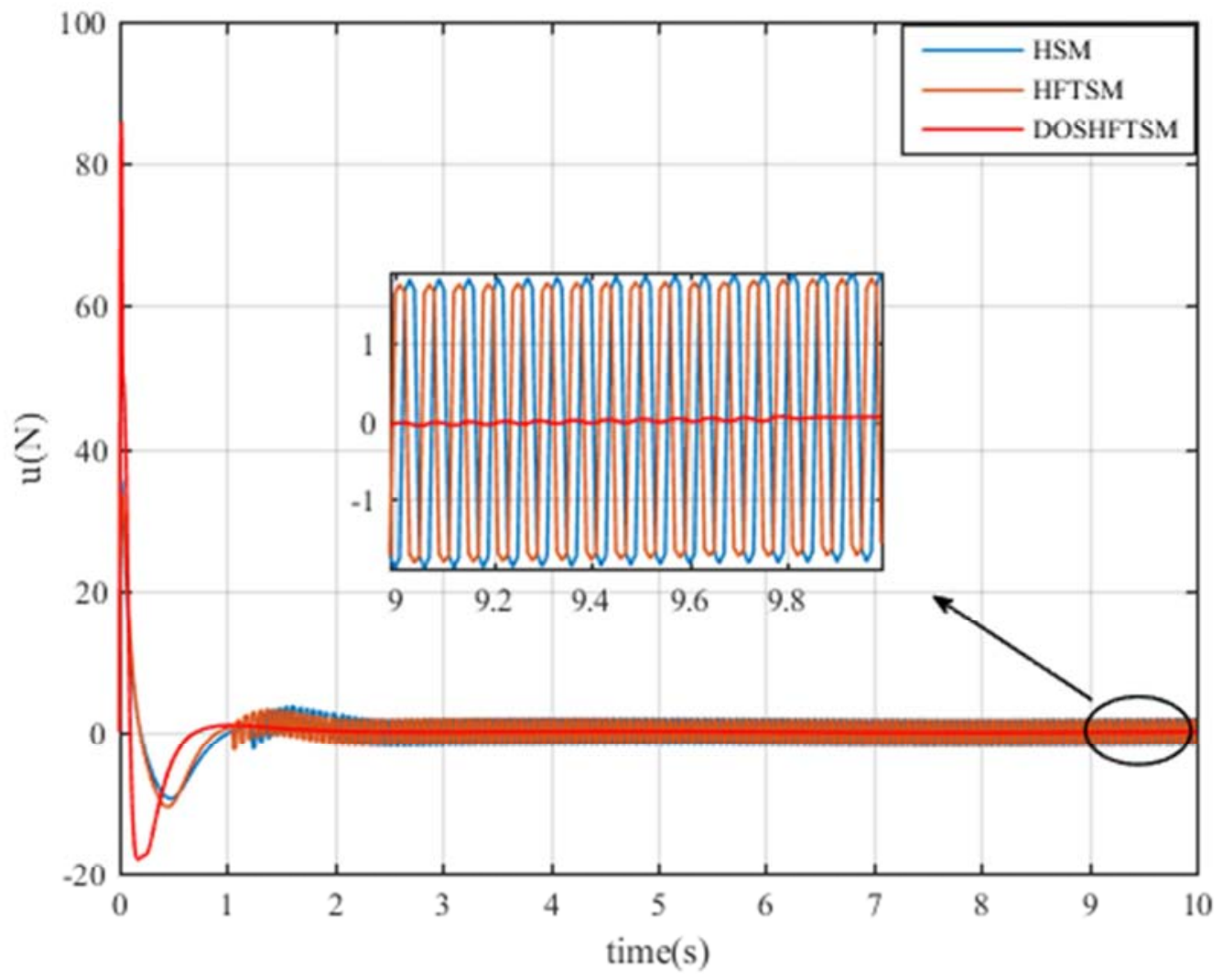

Figure 5. The response curves of the control input. 

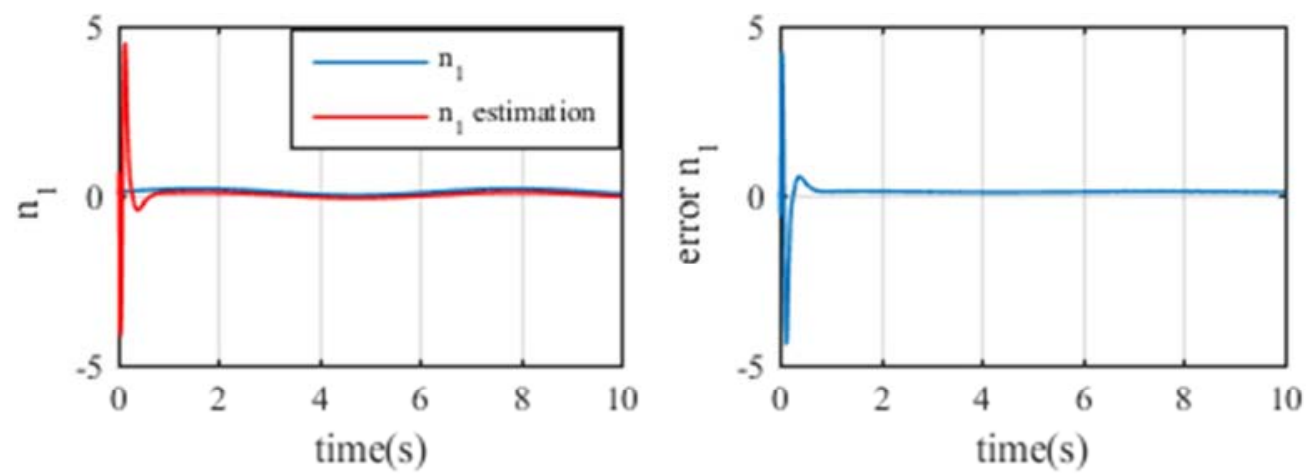

a). The tracking response curve of $n_{1}$.
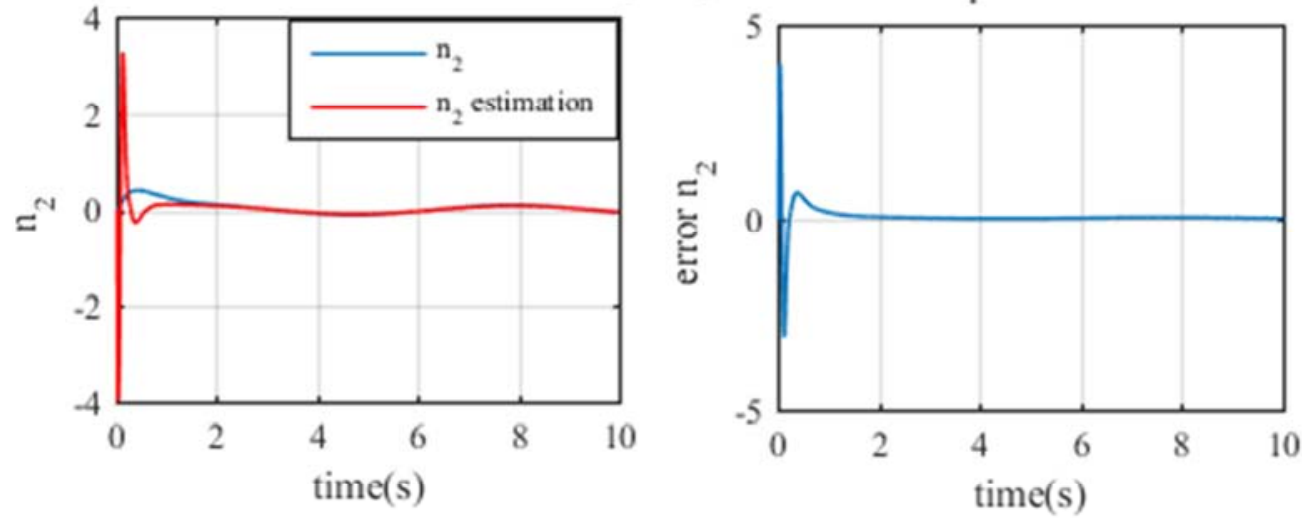

b). The tracking response curve of $n_{2}$.

Figure 6. The disturbance observation results of the disturbance observer.

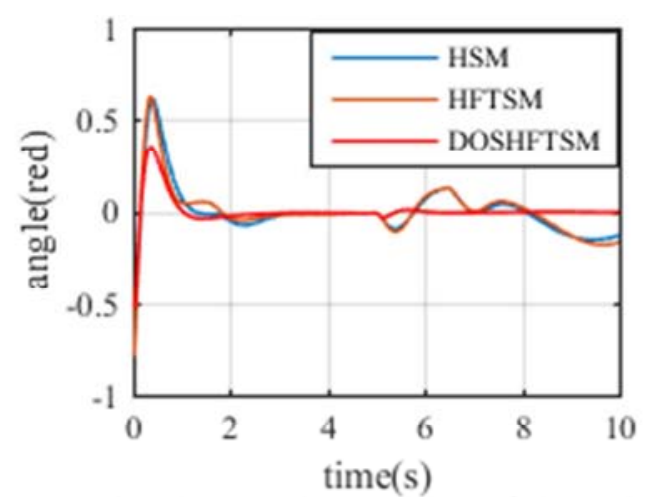

a). The angular position of the pole.

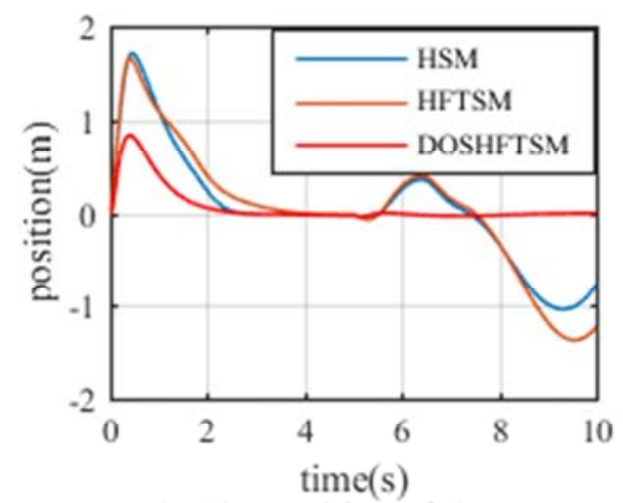

c). The position of the cart.

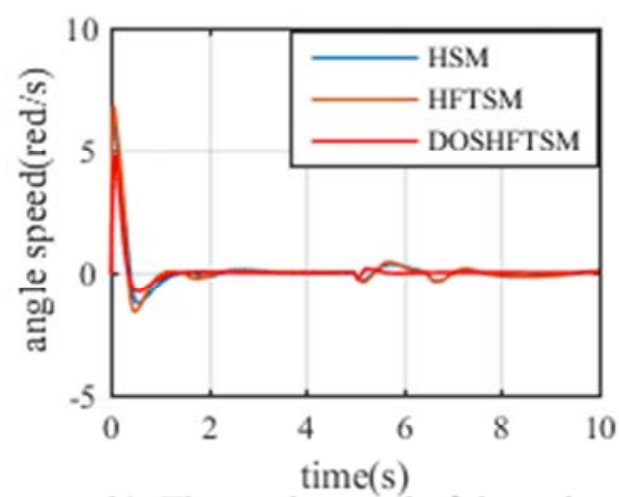

b). The angle speed of the pole.

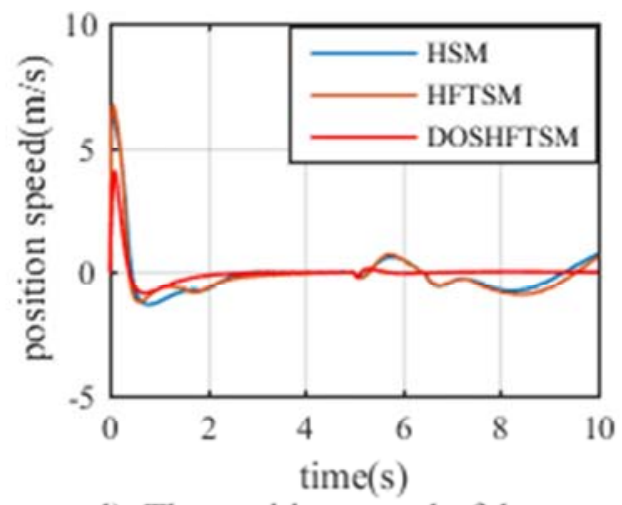

d). The position speed of the cart.

Figure 7. The response curves of the system state (with different conditions). 


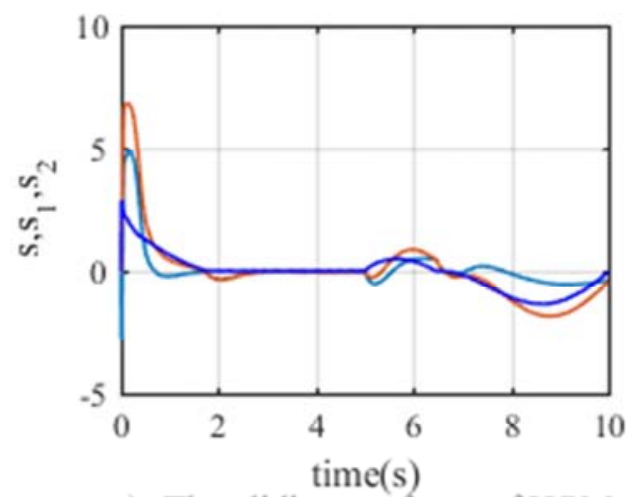

a). The sliding surfaces of HSM.

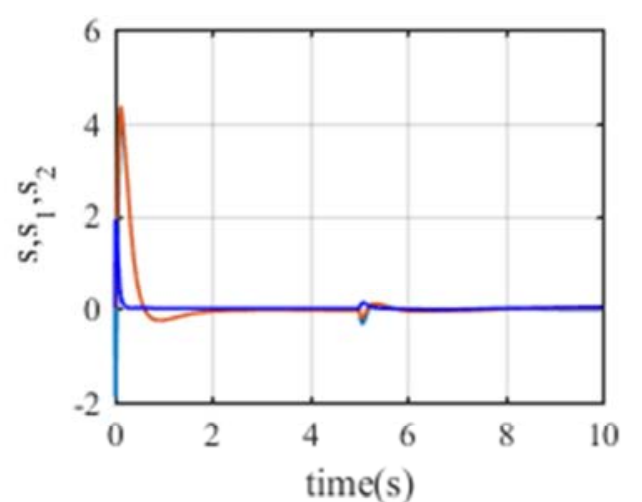

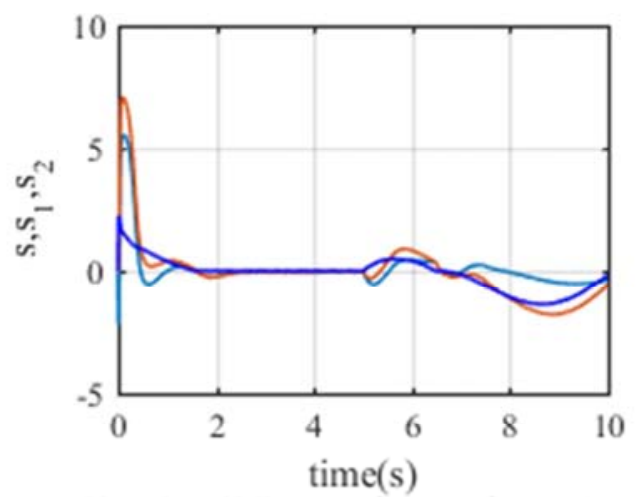

b). The sliding surfaces of HFTSM.

c). The sliding surfaces of DOSHFTSM.

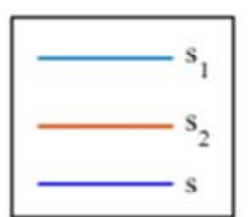

Figure 8. The response curves of the sliding surfaces (with different conditions).
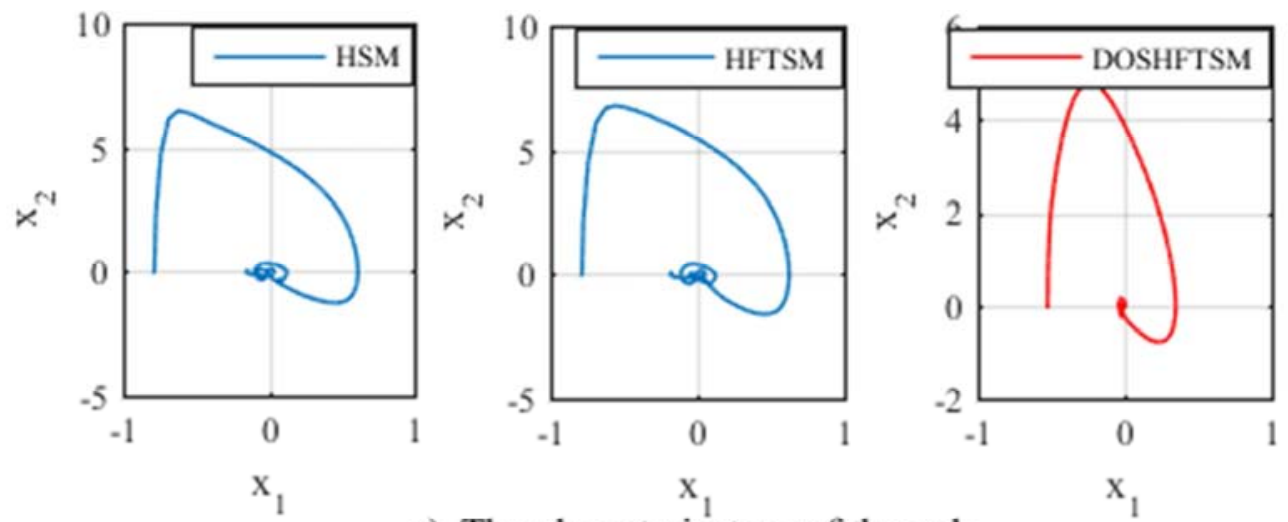

a). The phase trajectory of the pole
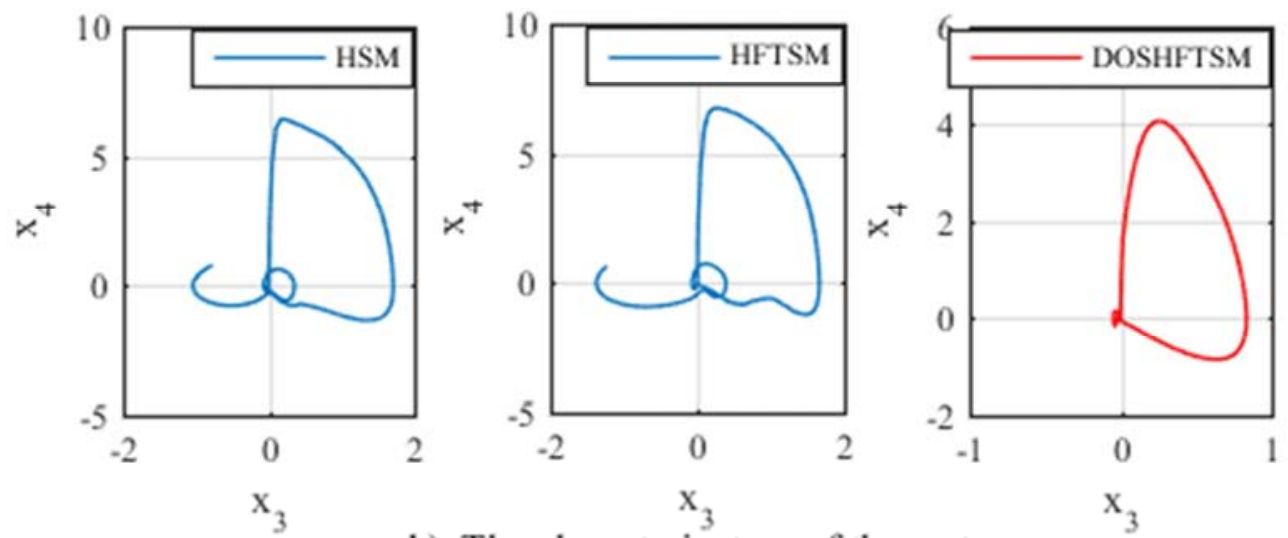

b). The phase trajectory of the cart.

Figure 9. The response curves of the system phase trajectory (with different conditions). 


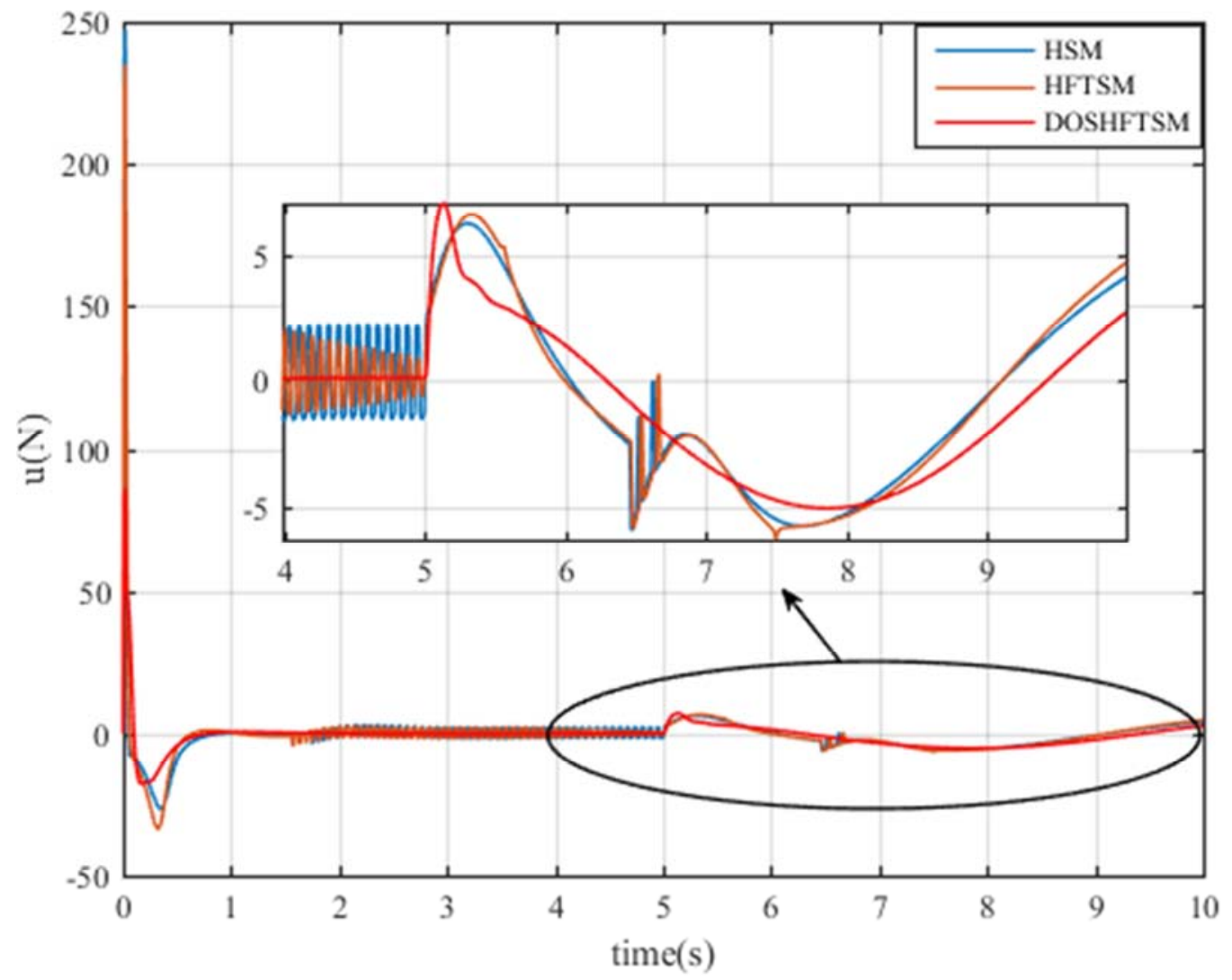

Figure 10. The response curves of the control input (with different conditions).

In this paper, the matlab real-time simulation tool and Simulink toolbox are used to test the designed controller on the actual vehicle system. Figure 11 shows the linear motor inverted pendulum system manufactured by Hopemotion Co., Ltd.

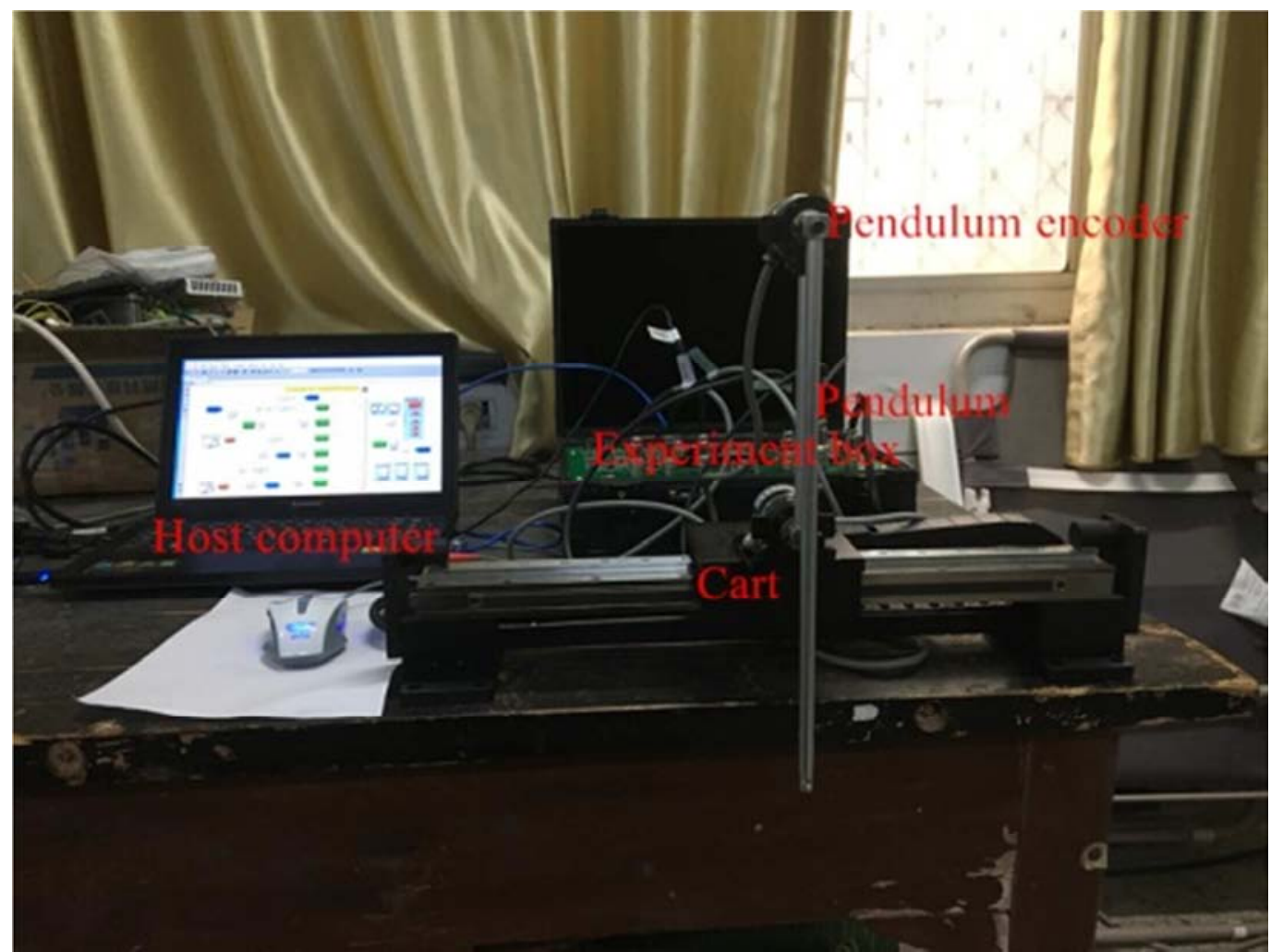

Figure 11. The practical cart-pole system. 


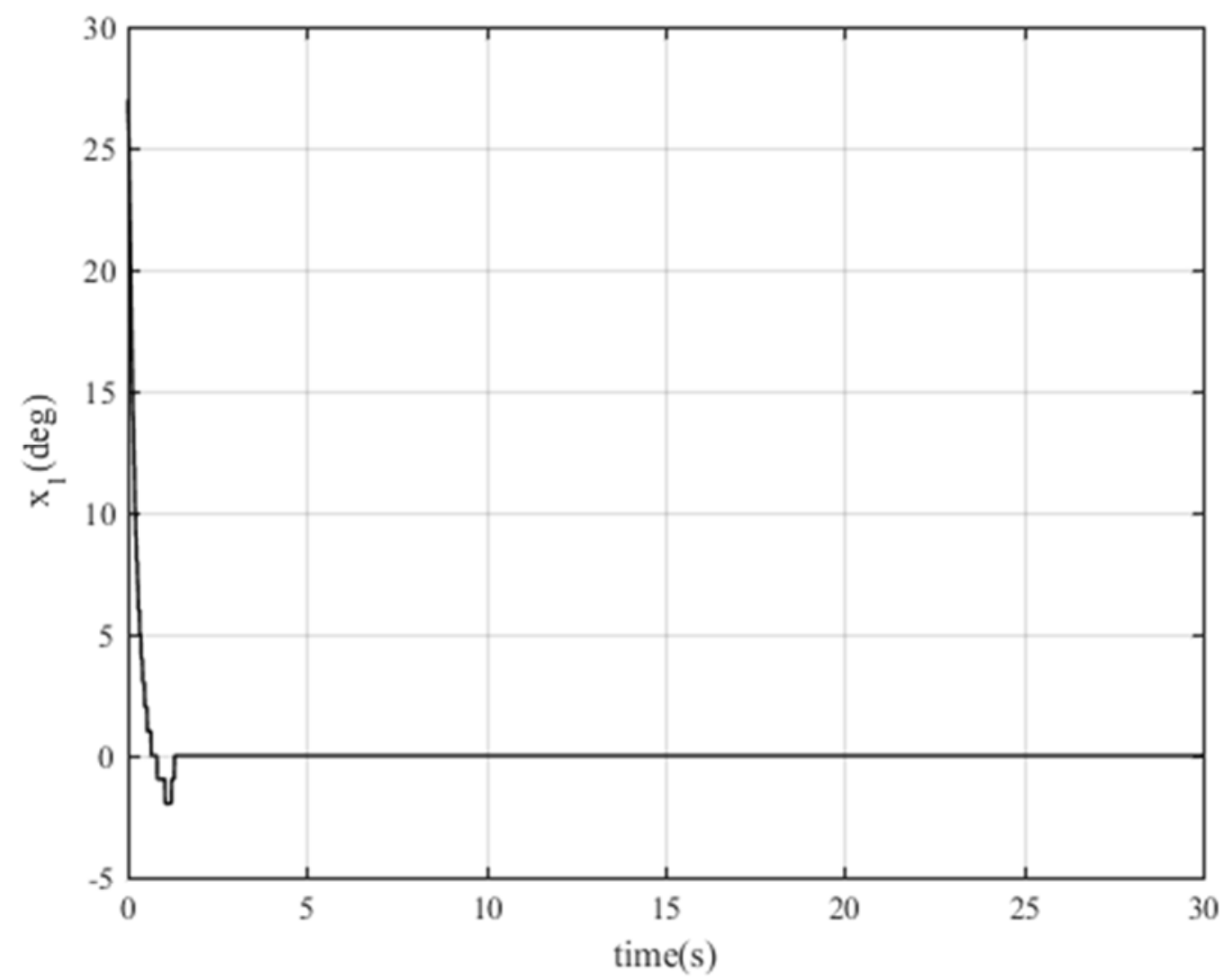

Figure 12. The response curves of the pendulum swing angle (physical simulation).

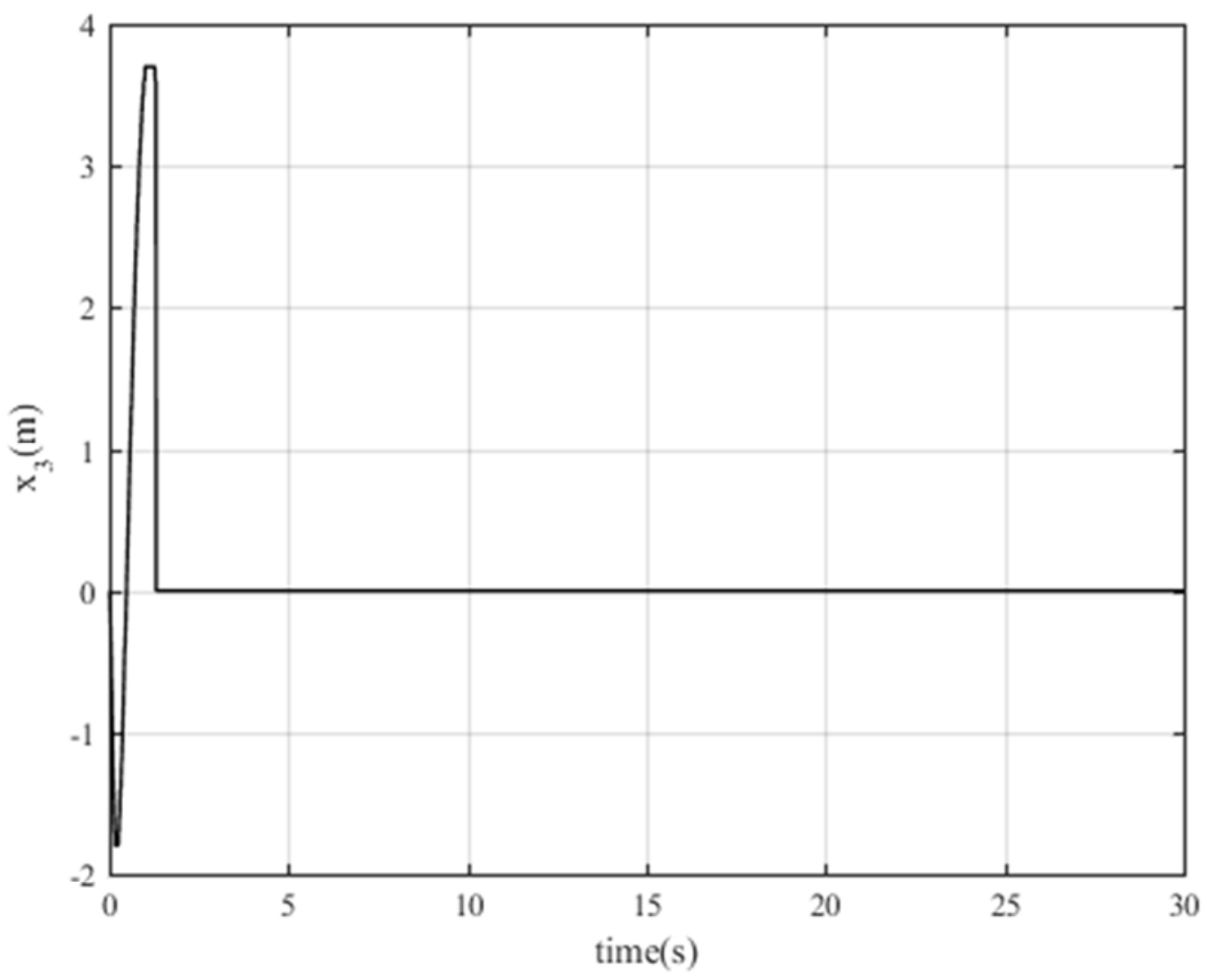

Figure 13. The response curves of the trolley position (physical simulation). 


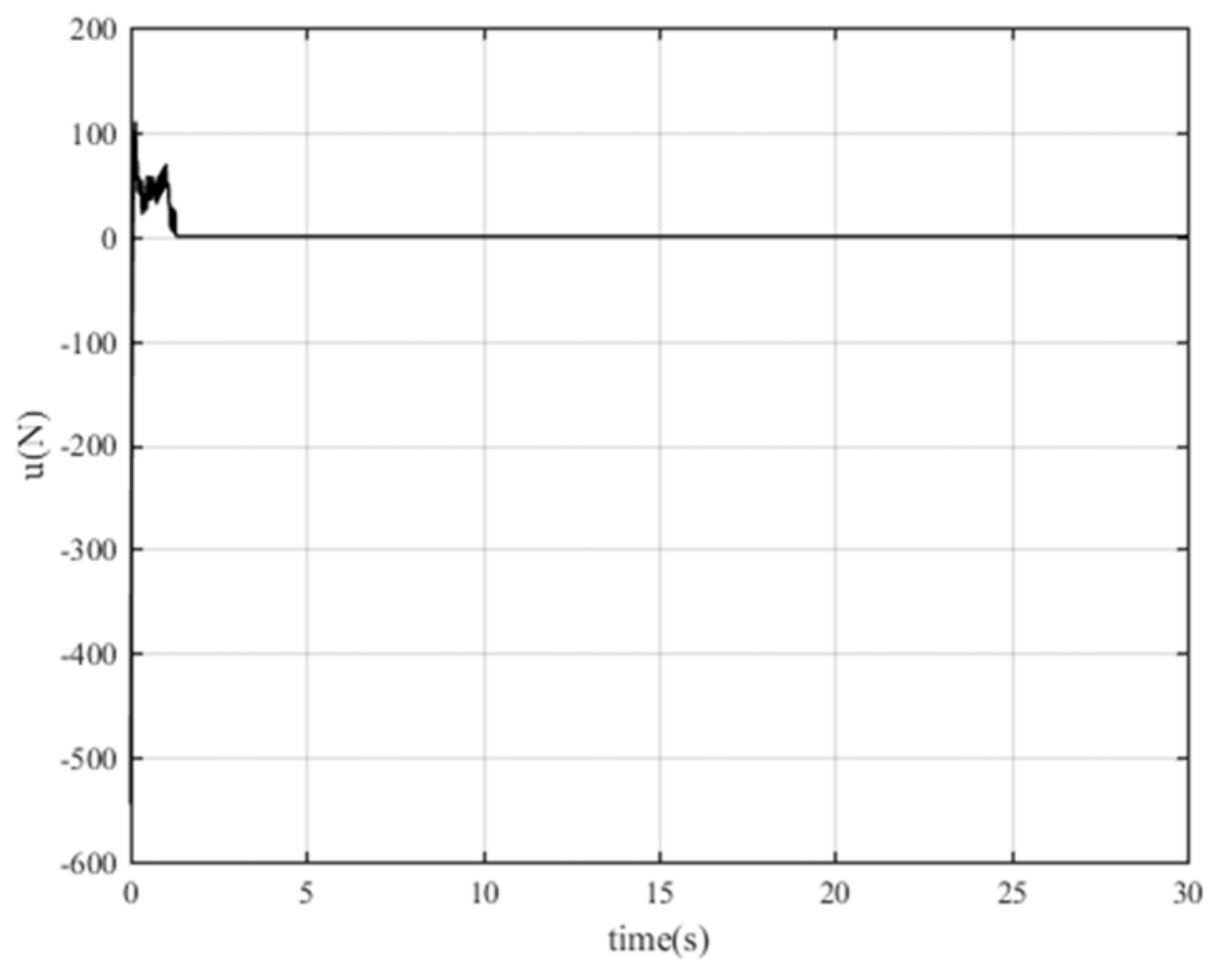

Figure 14. The response curves of the control input (physical simulation).

Figures 12-14 are the experimental curves of the pendulum swing angle, trolley position and control input, respectively. It can be clearly seen that the swing angle, position and control inputs can be quickly maintained stable. The results of physical experiments further validate the effectiveness of the scheme.

\section{Conclusions}

A second-order hierarchical fast terminal sliding mode control scheme based on disturbance observer is proposed for a class of fourth-order underactuated mechanical systems. Equivalent control terms and switching control terms of sliding mode controller are designed by using second-order hierarchical fast terminal sliding mode surface and variable coefficient double power reaching law. The tracking differentiator principle is used to design a hyperbolic tangent nonlinear disturbance observer. The uncertainties and external disturbances of the system are estimated, and the sliding mode controller is compensated to improve the robustness of the system. The stability of the system is verified by Lyapunov principle. Finally, the effectiveness of this method is verified by numerical simulation experiment and physical simulation of inverted pendulum. Therefore, the application of this method to the control of underactuated mechanical systems such as multi-stage inverted pendulum, manipulator system, torch system and spherical plate system deserves further study.

\section{Conflict of Interest Statement}

All the authors do not have any possible conflicts of interest.

\section{References}

[1] Reyhanoglu, M., Schaft, A. V. D., Mcclamroch, N. H., and Kolmanovsky, I. V. "Dynamics and control of a class of underactuated mechanical systems. "IEEE Transactions on Automatic Control 44.9 (2017): 1663-1671.

[2] She, J., Zhang, A., Lai, X., and Wu, M. "Global stabilization of 2-DOF underactuated mechanical systems - an equivalent-i nput-disturbance approach." Nonlinear Dynamics 69.1-2 (2012): 495-509.

[3] Sarras, I., Acosta, J. á., Ortega, R., and Mahindrakar, A. D. "Constructive immersion and invariance stabilization for a class of underactuated mechanical systems." Automatica 49.5 (2013): 1442-1448.

[4] Lu, Biao, Y. Fang, and N. Sun. "Continuous Sliding Mode Control Strategy for a Class of Nonlinear Underactuated Systems." IEEE Transactions on Automatic Control PP.99 (2018): 1-1.

[5] Liu. "Control of a class of multibody underactuated mechanical systems with discontinuous friction using slidingmode." Transactions of the Institute of Measurement and Control 40.2 (2016): 514-527.

[6] Yue, B. Y. Liu, C. An, and X. J. Sun. "Extended state observer-based adaptive hierarchical sliding mode control for longitudinal movement of a spherical robot." Nonlinear Dynamics 78.2 (2014.): 1233-1244.

[7] He, C. Zhang, N. Yang, and H. Li. "Exquisite disturbance attenuation control for a rotary inverted pendulum." Transactions of the Institute of Measurement and Control 40.3 (2018.): 812-818. 
[8] Wang, S. Li, and Q. W. Chen. "Robust adaptive finite-time tracking control of uncertain mechanical systems with input saturation and dead zone." Transactions of the Institute of Measurement and Control, 41.2 (2019): 560-572.

[9] Wang, J. Q. Yi, D. B. Zhao, and D. T. Liu. "Design of a stable sliding-mode controller for a class of second-order underactuated systems." IEE Proceedings-Control Theory and Applications 151.6 (2004): 683-690.

[10] Ning S, Wu Y, Fang Y, et al. Nonlinear Continuous Global Stabilization Control for Underactuated RTAC Systems: Design, Analysis, and Experimentation [J]. IEEE/ASME Transactions on Mechatronics, 2017, 22 (2): 1104-1115.

[11] Dian S, Chen L, Hoang S, et al. Gain scheduled dynamic surface control for a class of underactuated mechanical systems using neural network disturbance observer [J]. Neurocomputing, 2018, 275: S0925231217316934.

[12] Binh N T, Tung N A, Nam D P, et al. An Adaptive Backstepping Trajectory Tracking Control of a Tractor Trailer Wheeled Mobile Robot [J]. International Journal of Control Automation and Systems, 2019 (7): 1-9.

[13] Wang, J. Q. Yi, D. B. Zhao, and D. T. Liu. "Adaptive sliding mode controller for an underactuated manipulator." Proceedings of 2004 International Conference on Machine Learning and Cybernetics (IEEE Cat. No.04EX826), Shanghai, China, 2004, pp. 882-887 vol. 2.

[14] Almutairi, Naif B., and M. Zribi. "On the sliding mode control of a Ball on a Beam system." Nonlinear Dynamics 59.1-2 (2009): 221-238.

[15] Xu, Rong, and Özgüner, Ümit. "Sliding mode control of a class of underactuated systems." Automatica 44.1 (2008): 233-241.

[16] Xu, Jian Xin, Z. Q. Guo, and T. H. Lee. "Sliding mode controller design for underactuated systems." (2012).

[17] Musmade, and B. Patre. "Sliding mode control design for robust regulation of time-delay processes." Transactions of the Institute of Measurement and Control 37.6 (2014): 699-707.

[18] Lan, S. Li, J. Yang, and L. Guo. "Finite-time soft landing on asteroids using nonsingular terminal sliding mode control." Transactions of the Institute of Measurement and Control 36.2 (2013): 216-223.

[19] Tian, Zhixiang, and H. Wu. "Hierarchical Terminal Sliding Mode Control for Underactuated Space Robots." International Conference on Machine Vision \& Human-machine Interface IEEE, 2010.

[20] Lee, J. B. Park, and Y. H. Choi. "Finite time control of nonlinear underactuated systems using terminal sliding surface." IEEE International Symposium on Industrial Electronics (2009) pp. 626-631.

[21] Wang, Y. Wu, E. Zhang, J. Guo, and Q. Chen. "Adaptive terminal sliding-mode controller based on characteristic model for gear transmission servo systems. Transactions of the Institute of Measurement and Control 41.1 (2018): 219-234.
[22] Zhu, Qidan Zhu Qidan, R. Y. R. Yu, and Z. L. Z. Liu. "A discontinuous control law of an underactuated surface vessel based on fast terminal sliding mode." World Congress on Intelligent Control \& Automation IEEE, 2010.

[23] Yu, and X. Huo. "Fast terminal sliding-mode control design for nonlinear dynamical systems." IEEE Transactions on Circuits \& Systems I Fundamental Theory \& Applications 49.2 (2009): 261-264.

[24] Zheng, Naijia, et al. "Hierarchical fast terminal sliding mode control for a self-balancing two-wheeled robot on uneven terrains." Control Conference IEEE, 2017.

[25] Boukattaya M, Mezghani N, Damak T. Adaptive nonsingular fast terminal sliding-mode control for the tracking problem of uncertain dynamical systems. [J]. Isa Transactions, 2018, 77: S0019057818301538.

[26] Pai M C. Synchronization of unified chaotic systems via adaptive nonsingular fast terminal sliding mode control $[\mathrm{J}]$. International Journal of Dynamics and Control, 2018.

[27] Haibo L, Heping W, Junlei S. Attitude control for QTR using exponential nonsingular terminal sliding mode control [J]. Journal of Systems Engineering and Electronics, 2019, 30 (1): 191-200.

[28] Ding, Feng, et al. "Sliding mode control with an extended disturbance observer for a class of underactuated system in cascaded form." Nonlinear Dynamics (2017).

[29] Yue, X. Wei, and Z. Li. "Zero-dynamics-based adaptive sliding mode control for a wheeled inverted pendulum with parametric friction and uncertain dynamics compensation." Transactions of the Institute of Measurement and Control 37.1 (2014): 91-99.

[30] Jouini, Marwa, S. Dhahri, and A. Sellami. "Combination of integral sliding mode control design with optimal feedback control for nonlinear uncertain systems." Transactions of the Institute of Measurement and Control (2018).

[31] Wang, J. Yuan, and Y. Pan. "Adaptive second-order sliding mode control: A unified method." Transactions of the Institute of Measurement and Control 40.6 (2017): 1927-1935.

[32] Lo, Ji Chang, and Y. H. Kuo. "Decoupled fuzzy sliding-mode control." IEEE Transactions on Fuzzy Systems 6.3 (1998): 426-435.

[33] Han. "nonlinear tracking-differentiator." Journal of Systems Science and Mathematical Sciences 14.2 (1994).

[34] Mao, Wei. Li, and X. L. Feng. "Nonlinear tracking differentiator design based on hyperbolic tangent." Computer applications 36. zl (2016).

[35] Marks, G., et al. "Effects of High Order Sliding Mode Guidance and Observers On Hit-to-Kill Interceptions." Aiaa Guidance, Navigation, \& Control Conference \& Exhibit 2006.

[36] Wang, W., et al. "Design of a stable sliding-mode controller for a class of second-order underactuated systems." IEE Proceedings-Control Theory and Applications 151.6 (2004): 683-0. 